\title{
Lung abscess due to Streptococcus pneumoniae simulating pulmonary tuberculosis: presentation of two cases
}

\author{
Alessandro Perazzo, ${ }^{1}$ Piergiorgio Gatto, ${ }^{1}$ Gianluca Ferraioli, ${ }^{2}$ Renata Senarega,${ }^{3}$ Cornelius Barlascini, ${ }^{4}$ Mario Santo, ${ }^{5}$ \\ Silvia Reali, ${ }^{6}$ Antonello Nicolini ${ }^{1}$ \\ ${ }^{1}$ Respiratory Diseases Unit, General Hospital, Sestri Levante (GE); ${ }^{2}$ Emergency Department ASL4 Chiavarese (GE); \\ ${ }^{3}$ Radiology Department ASL4 Chiavarese (GE); ${ }^{4}$ Forensic Medicine ASL4 Chiavarese (GE); ${ }^{5}$ Emergency Medicine General \\ Hospital, Lavagna (GE); ${ }^{6}$ Microbiology Service, General Hospital, Lavagna (GE), Italy
}

\begin{abstract}
In the past, anaerobes were the most common cause of community-acquired lung abscess; Streptococcus species were the second most common cause. In recent years, this has changed. Klebsiella pneumoniae is now most common cause of community-acquired lung abscess, although Streptococcus species remain pathogen of major importance. We present two cases of pulmonary cavitation due to Streptococcus pneumoniae which resembled pulmonary tuberculosis with regards to their history and radiological findings. These are examples of a common diagnosis presenting in an uncommon way. Our cases had some peculiarities: they had a clinical picture strongly suggestive of pulmonary tuberculosis or lung cancer rather than necrotizing infectious pneumonia in patients with no comorbidities or underlying diseases (including oral or dental pathologies). Radiological findings did not help the clinicians: pulmonary tuberculosis was the first diagnostic hypothesis in both cases. An underlying lung cancer was excluded in the first case only after invasive pulmonary procedures.
\end{abstract}

\section{Introduction}

Prior to the introduction of antimicrobials into clinical practice, one third of patients with a lung abscess died. Antimicrobial use has improved the prognosis of this condition, but the mortality rate was estimated to

Correspondence: Nicolini Antonello, Respiratory Diseases Unit, General Hospital, via Terzi 43, 16039 Sestri Levante (GE), Italy.

Tel.: +39.018.5329145 - Fax: +39.018.5329935.

E-mail: antonello.nicolini@fastwebnet.it

Key words: lung abscess, Streptococcus pneumoniae, pulmonary tuberculosis.

Contributions: AP, reviewed the cases, drafted and revised the manuscript; PG, GF, and CB, revised the manuscript; RS, MS, and SR, reviewed the cases and the manuscript; $\mathrm{AN}$, drafted and revised the manuscript.

Conflict of interests: the authors declare that they have no potential conflict of interests.

Received for publication: 19 February 2013.

Revision received: 9 March 2013.

Accepted for publication: 11 May 2013.

This work is licensed under a Creative Commons Attribution NonCommercial 3.0 License (CC BY-NC 3.0).

(C) Copyright A. Perazzo et al., 2014

Licensee PAGEPress, Italy

Italian Journal of Medicine 2014; 8:39-43

doi:10.4081/itjm.2014.103 be as high as $38.2 \%$ in reports published between 1969 and 2005. Lung abscess remains an important pathological entity. Anaerobes and microaerophilic streptococci are responsible for $60-80 \%$ of lung abscesses $;{ }^{1}$ Streptococcus pneumoniae is a quite uncommon cause of lung abscess. The aim of this report is to present two cases simulating pulmonary tuberculosis which challenged clinicians.

\section{Case Reports}

\section{Case \#1}

A 45-year old man with a past medical history that included only intermittent bronchial asthma was admitted to hospital with a 2-month history of productive cough, low grade fever, intermittent chest pain, asthenia and anorexia with accompanying weight loss $\mathrm{He}$ also complained of hemoptysis which had started 2 days prior to his admission. He was neither a smoker nor a drinker.

On examination, the patient appeared ill: his temperature was $37.2^{\circ} \mathrm{C}$; heart rate 78 beats per minute; respiratory rate 18 breaths per minute; arterial blood pressure $105 / 70 \mathrm{mmHg}$, and oxygen saturation $99 \%$ on room air. Bronchial breath sounds were noted over the area of the right upper lung along with scattered crackles.

Laboratory investigations demonstrated a white blood cell count of 8,540 cells $/ \mu \mathrm{L}$, with $74.5 \%$ neutrophils and $13.0 \%$ lymphocytes, a red blood cell count of 41,770,000 cells $/ \mu \mathrm{L}$, hemoglobin $14.0 \mathrm{~g} / \mathrm{dL}$, 
platelet count 283,000 cells $/ \mu \mathrm{L}, \mathrm{C}$-reactive protein $3.51 \mathrm{mg} / \mathrm{dL}$, lactate dehydrogenase $255 \mathrm{U} / \mathrm{L}$, and total protein $7.44 \mathrm{~g} / \mathrm{dL}$ with $17.0 \% \gamma$-globulins. A chest radiograph showed right upper excavation (Figure 1A). Because of the suspicion of infection by Mycobacterium tuberculosis, the patient was admitted to a negative pressure room in the Respiratory Disease Unit. Computed tomography (CT) of the chest showed a cavitary pulmonary nodule of $6.7 \times 4.6 \mathrm{~cm}$ at the level of the dorsal segment of the right upper lobe (Figure 1B).

Flexible bronchoscopy revealed bronchial edema with narrowing of the right superior lobe bronchus. Moreover, a small amount of purulent secretion was seen. A cytological examination showed inflammatory cells but no malignancy. Direct microscopy and bacteriological analysis performed on broncho-alveolar lavage fluid and bronchoaspirate culture, including polymerase chain reaction analysis, were negative for Mycobacterium tuberculosis.

Because of the suspicion of an underlying malignancy, a CT-guided biopsy was performed in the right upper lobe. The cytological examination showed inflammatory cells compatible with active inflammation, but no malignant cells were seen. A Gram-stained
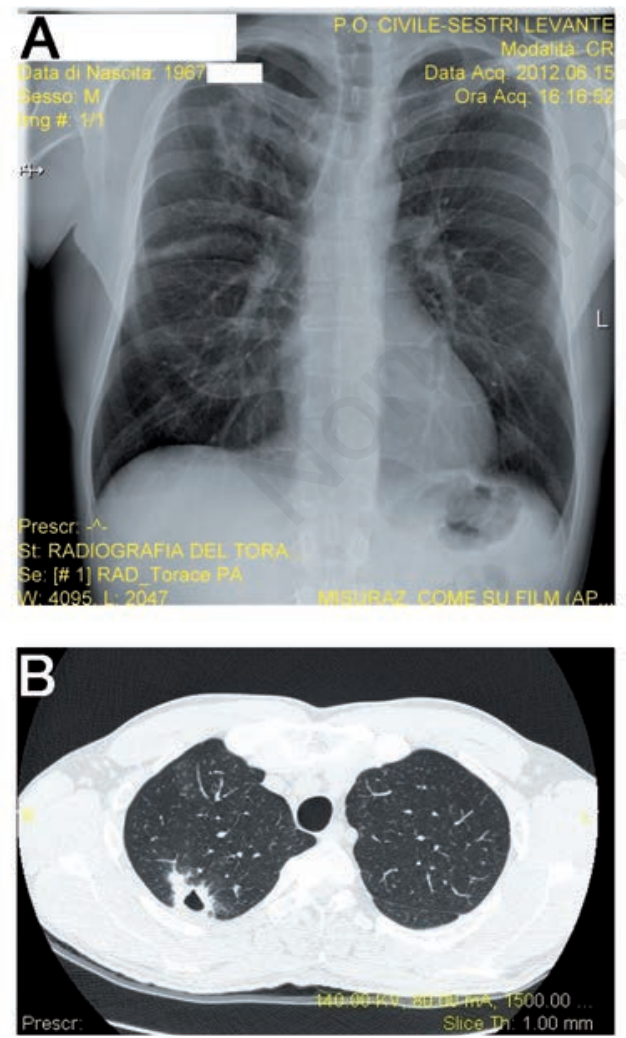

Figure 1. A) Chest radiograph showing a fluid-filled cavity in the right upper lobe; B) Chest computed tomography showing a spherical mass with an air-fluid level in the right upper pulmonary lobe. smear of sputum revealed numerous polymorphonuclear neutrophils and Gram-positive cocci in pairs and chains; the culture eventually yielded Streptococcus pneumoniae. All the blood cultures collected at admission were negative, as was the Streptococcus pneumoniae urinary antigen. A dental radiograph did not show infectious foci.

The minimum inhibitory concentration breakpoints for penicillin G, ceftriaxone, erythromycin and imipenem were $\leq 0.06, \leq 0.06, \geq 0.5$, and $\leq 0.03$, respectively. The biochemical and susceptibility profile of these strains were assayed using an automated system for identification and susceptibility tests (ViteK Bio Merieux SA, Marcy l'Étoile, France). The criteria for defining susceptibility or lack of susceptibility were based on the Eucast guidelines (http://www.eucast.org/clinical_breakpoints).

The patient was treated with amoxicillin/clavulanate $2000 \mathrm{mg} / 200 \mathrm{mg}$ i.v. every $8 \mathrm{~h}$ for three weeks. The patient continued treatment with amoxicillin/clavulanate ( $875 \mathrm{mg} / 125 \mathrm{mg}$ tablets every 8 hours) for 2 weeks after his discharge from hospital. After 6 weeks chest CT showed complete resolution of the lung abscess.

\section{Case \#2}

A 27-year old man who reported having no previous diseases was admitted to hospital with a 1month history of productive cough, light fever, asthenia, anorexia, and severe weight loss $(30 \mathrm{~kg})$. He did not smoke or drink alcohol. On admission his temperature was normal $\left(36.5^{\circ} \mathrm{C}\right)$. In the subsequent clinical examination, the patient appeared ill but not toxic, and his temperature was $37.2^{\circ} \mathrm{C}$, heart rate 98 beats per minute, respiratory rate 18 breaths per minute, blood pressure 100/66 $\mathrm{mmHg}$, and oxygen saturation $97 \%$ in room air. Bronchial breath sounds were noted over the distribution of the right upper lung along with scattered crackles.

Laboratory evaluation demonstrated a white blood cell count of 10,700 cells $/ \mu \mathrm{L}$, with $75.5 \%$ neutrophils, $14.1 \%$ lymphocytes, a red blood cell count of 4,140,000 cells $/ \mu \mathrm{L}$, hemoglobin level of $12.4 \mathrm{~g} / \mathrm{dL}$, platelet count of 241,000 cells $/ \mu \mathrm{L}, \mathrm{C}$-reactive protein $8.05 \mathrm{mg} / \mathrm{dL}$, lactate dehydrogenase $201 \mathrm{U} / \mathrm{L}$, and total proteins 7.31 $\mathrm{g} / \mathrm{dL}$ with $15.1 \% \gamma$-globulins.

A chest radiograph showed right upper lobe excavation (Figure 2A). Because of the suspicion of an infection due to Mycobacterium tuberculosis the patient was admitted to a negative pressure room in the Respiratory Disease Unit. Chest CT showed a cavitary pulmonary nodule of $10.7 \times 7.9 \mathrm{~cm}$ at the level of the dorsal segment of the right upper lobe (Figure 2B).

The patient underwent fiber-bronchoscopy which showed bronchial edema with narrowing of the superior lobe bronchus and a small amount of purulent secretion. A cytological examination showed inflammatory cells, 
but no malignancy. A bronchial biopsy was performed: the histopathological examination showed inflammation, but no malignancy.

Direct microscopy and bacteriological analysis and polymerase chain reaction analysis performed on broncho-alveolar lavage fluid and culture of bronchoaspirate were negative for Mycobacterium tuberculosis. A Gram-stained smear of bronchial aspirate revealed numerous polymorphonuclear neutrophils and Grampositive cocci in pairs and chains; the culture eventually yielded Streptococcus pneumoniae. All the blood cultures collected on admission were negative, as was Streptococcus pneumoniae urinary antigen.

The minimum inhibitory concentration breakpoints of penicillin G, ceftriaxone, erythromycin and imipenem were $\leq 0.06, \leq 0.06, \geq 0.5$, and $\leq 0.03$, respectively. The biochemical and susceptibility profiles of the strains were determined using an automated system for identification and susceptibility tests (Viteliz Bio Merieux SA). The criteria for define susceptibility
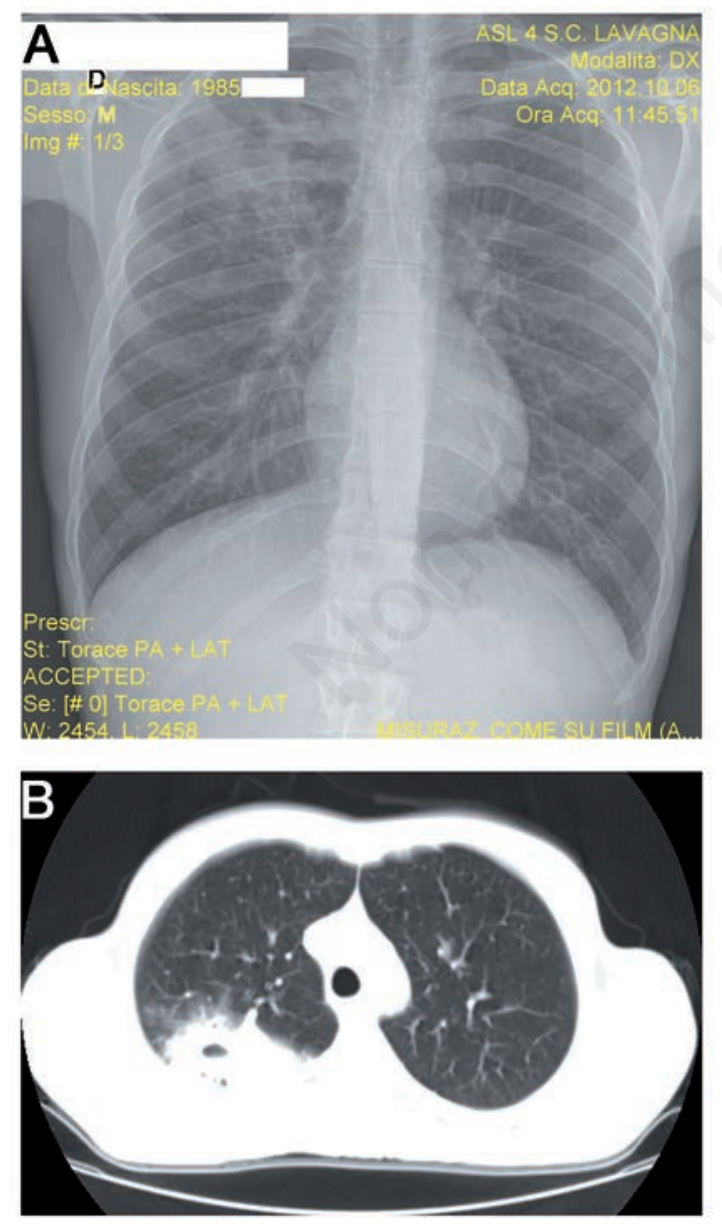

Figure 2. A) Chest radiograph showing a cavitation in the right upper lobe; B) Chest computed tomography showing a spherical mass with cavitation in the right upper pulmonary lobe. or lack of susceptibility were based on the Eucast guidelines.

A dental radiograph did not show infectious foci. The patient was treated with amoxicillin/clavulanate 2 g every $8 \mathrm{~h}$ for 3 weeks and then with amoxicillin/clavulanate $1 \mathrm{~g}$ every $8 \mathrm{~h}$ for a further 3 weeks after discharge from hospital. After 8 weeks a chest CT showed complete resolution of the lung abscess.

Both patients gave consent to the publication of material relating to them.

\section{Discussion}

In the past, anaerobes were the most common cause of community-acquired lung abscess. Streptococcus species were the second most common cause of this problem. In recent years, this has changed. Klebsiella pneumoniae is now one of the most common cause of community-acquired lung abscess, ${ }^{1}$ although Streptococcus species remain important pathogens.

In a recent retrospective review of 205 patients, Takanayagi et al. documented 122 bacteriological results, with the isolation of 189 bacterial species. ${ }^{1}$ Pure aerobic, mixed aerobic and anaerobic, and pure anaerobic bacteria were isolated in 90 (73.8\%), 17 (13.9\%), and $15(12.3 \%)$ patients, respectively. The four most common etiologic pathogens were Streptococcus species (59.8\%), anaerobes (26.2\%), Gemella species (9.8\%), and Klebsiella pneumoniae (8.2\%). Streptococcus mitis was the most streptococcus. Streptococcus pneumoniae was found in three patients $(2.12 \%){ }^{1}$

Necrotizing pneumonia has generally been regarded as a rare complication of pneumococcal infection in adults. ${ }^{2,3}$ Type 3 Streptococcus pneumoniae was the single most common type implicated in necrosis; however, many other serotypes have been implicated. ${ }^{2}$ Necrotizing pneumonia predominately affects children, although it can occur in adults. ${ }^{2}$ In the USA and in Taiwan, infection with Streptococcus pneumoniae serotype 3 (mainly ST180) was a significant contributor to necrotizing pneumonia. In Taiwan, serotype 14 was another common serotype that caused necrotizing pneumonia. However, there is no monopoly among the serotypes; other serotypes including 6B, 14, 19F, $23 \mathrm{~F}$ and $19 \mathrm{~A}$, at a lower frequency, may also cause necrotizing pneumonia. ${ }^{4}$

In a retrospective review conducted by Yen et al., 5 covering 20 years (1982-2002), 23 patients presented with a lung abscess; the most common micro-organism isolated was Streptococcus pneumoniae. ${ }^{5}$ Currently 46 serogroups and 93 serotypes have been documented. Serotype 6C, serotype 6D, and serotype 11E1-3 have been the latest additions. Severe disease is more frequent in individuals at extremes of age and those with immunological impairment (children or adults). A significant proportion of adults who develop 
an invasive infection have no apparent pre-existing risk factor. Most cases of severe pneumococcal disease are caused by a limited number of serotypes that vary in infectivity and virulence. Invasive diseases caused by pneumococcus include meningitis, bacteremia, and pneumonia with bacteremia, lung abscess and/or empyema. Host and bacterial factors both contribute to the pathogenicity. The coincidence of two key situations may lead to the development of disease (first, colonization of the host with a pneumococcal serotype that the host has not yet established immunity to, and second, an alteration of the natural barriers or host immune system). ${ }^{2,5}$

$S$. pneumoniae frequently colonizes the nasopharyngeal niche; spread from the nasopharynx to the lower respiratory tract or other sites may cause invasive disease. The nasopharyngeal carriage rate is much higher in children, particularly during the first years of life (nasopharyngeal carriage rates from 20\% to $50 \%$ in healthy children) than in adults (nasopharyngeal carriage rates range from 5\% to $30 \%$ in healthy adults). Risk factors for nasopharyngeal carriage in children include winter season, age $<6$ years, having young siblings, and attending day care centers. In adults, risk factors for nasopharyngeal carriage include cigarette smoking, asthma, and acute upper respiratory infection. In children, colonization may persist for a mean of 4 months but is much shorter in adults, usually 2 to 4 weeks. ${ }^{6,7}$ Transmission of pneumococcus from children to household contacts or adults is the principal cause of nasopharyngeal carriage and invasive pneumococcal diseases. Not all pneumococcal serotypes are equally capable of causing disease; the capsular polysaccharide is the major virulence factor, and those serotypes that produce large amounts of polysaccharide are likely to be more virulent than others. After invasion, the capsular polysaccharide also protects the pathogen by inhibiting neutrophil phagocytosis and classic complement-mediated bacterial killing.

Some of the important factors in the development of pneumococcal disease include the invasive properties of the serotype, the ability of the organism to evade the immune system, and the absence of a typespecific pneumococcal antibody. Ethnicity (AfricanAmericans, American Indians, native Alaskans, and Australian Aborigines), extremes of age $(<2$ or $>65$ years), existence of comorbidities (pulmonary, neurological, hepatic, diabetes mellitus, renal insufficiency or nephrotic syndrome), alcoholism, immunosuppression (asplenia, human immunodeficiency virus infection, and sickle cell disease), and anatomical abnormalities (cerebrospinal fluid leak, cochlear implant or congenital heart disease) are well-known risk factors associated with an increased susceptibility to invasive pneumococcal diseases and are associated with higher mortality. Exposure to cigarette smoke and multiple children in the household are the risk factors in healthy children. In the case of immunocompetent adults the incidence of severe pneumococcal disease is increased among subjects with the following comorbidities: congestive heart failure, chronic lung disease, asthma, diabetes mellitus, and neurological disorders. Alcohol abuse, cigarette smoking, recent influenza infection, institutionalization, male sex, and black race are also risk factors.

A lung abscess is an area of suppuration and necrosis involving one or more areas of the lung parenchyma. ${ }^{8} \mathrm{Abscesses}$ form as a consequence of infection and destruction of the lung parenchyma with central necrosis, leading to cavity formation. The characteristic radiographic finding is an air-fluid level on the chest X-ray. ${ }^{8,9} \mathrm{~A}$ lung abscess may decompress and eventually resolve, if it communicates with the tracheo-bronchial tree; however, if no communication develops, the capsule surrounding the necrotic lung and purulent material becomes thick and fibrotic.

Lung abscesses can result from widely variable pathogenic processes, including necrotizing pneumonia, aspiration pneumonia, focal infection of the lung during high-grade bacteremia, as a consequence of septic emboli or subacute airway infection. Conditions contributing to this pathogenic process include cystic fibrosis, tracheoesophageal fistula, gastroesophageal reflux, immunodeficiency, alcohol or drug use, acute and chronic aspiration and poor dental care ${ }^{8}$ Necrotizing community-acquired pneumonia is the most common underlying condition (particularly in children) with lung abscess. ${ }^{5,8}$

Lung abscesses can be classified in two groups: i) primary lung abscesses, which occur in patients without other underlying disease processes, that is, as a direct result of pneumonia or aspiration; ii) secondary lung abscesses, which develop in patients with an underlying medical condition such as an immunocompromised state, other lung disease, central nervous system disorder and congenital heart disease. The abscesses are located predominantly in posterior segments of the upper lobes or in superior segments of the lower lobes. They are typically singular and appear with equal frequency on the right or left side. Multiple or bilateral abscesses are rarely caused by aspiration: when present pathogenic mechanisms other than aspiration (for example, an extrapulmonary focus) should be suspected. ${ }^{10,11}$

The presence of pericavitary lesions and foci of pulmonary consolidation located far from a cavitary lesion suggest tuberculosis. Both clinical examination and postero-anterior coupled with lateral chest X-rays frequently clearly identify the characteristics and location of these lesions. Air-fluid levels can be seen within the cavities. ${ }^{11}$ Chest $\mathrm{CT}$ and endoscopic proce- 
dures are important in the analysis of the condition of the drainage bronchus and the wall of the abscess cavity, especially for making a differential diagnosis from lung carcinoma. ${ }^{11} \mathrm{~A}$ high number of patients undergo bronchoscopy despite current recommendations suggesting that this invasive technique should only be used in atypical cases and/or in those with a high degree of suspicious of mechanical obstruction. ${ }^{12,13}$ The duration of antibiotic administration in previous reports was estimated to be from 28 to 48 days, which is consistent with our usage. ${ }^{1,12,14,15}$

Clinicians should be aware that the differential diagnosis of pulmonary abscess includes bronchial neoplasms, either as necrotizing carcinoma or as the cause of post-stenotic cavernous pneumonia, other infectious diseases such as tuberculosis, Pneumocystis carinii pneumonia or endocarditis with septic metastases, and lung artery embolism or vasculitis (Wegener's disease). Fiberoptic bronchoscopy can be helpful in determining the cause of the disease, as may be CT-guided lung biopsy, or ultrasound-guided lung biopsy in the case of a large cavitated nodule.

\section{References}

1. Takayanagi N, Kagiyama N, Ishiguro T, et al. Etiology and outcome of community-acquired lung abscess. Respiration 2010;80:98-105.

2. Pande A, Nasir S, Rueda AM, et al. The incidence of necrotizing changes in adults with pneumococcal pneumonia. Clin Infect Dis 2012;54:10-6.

3. Yangco BG, Deresinski SC. Necrotizing or cavitating pneumonia due to Streptococcus pneumoniae: report of four cases and review of the literature. Medicine (Baltimore) 1980;59:449-57.
4. Janapatla RP, Hsu MH, Hsieh YC, et al. Necrotizing pneumonia caused by nanC-carrying serotypes is associated with pneumococcal haemolytic uraemic syndrome in children. Clin Microbiol Infect 2013;19:480-6.

5. Yen CC, Tang RB, Cheng SJ, Chin JW. Pediatric lung abscess: a retrospective review of 23 cases. J Microbiol Immunol Infect 2004;37:45-9.

6. Hodina M, Hanquinet S, Cotting J, et al. Imaging of cavitary necrosis in complicated childhood pneumonia. Eur Radiol 2002;12:391-6.

7. Boloorsaz MR, Khalizadeh S, Niknejad A, et al. Lung abscess in children: a 12-year study in national research institute of tuberculosis and lung disease. Tanaffos 2004;3:27-31.

8. Sethi S, Tolan RW. Multiple lung abscesses in a toddler. Hosp Phys 2008;44:17-22.

9. Chan PC, Huang LM, Wu PS. Clinical management and outcome of childhood lung abscess: a 16-year experience. J Microbiol Immunol Infect 2005;38:183-8.

10. Boloorsaz MR, Khalizadeh S, Sadeghi SM. Pediatric lung abscess: a retrospective review of 22 cases. Iran J Ped Soc 2007;1:19-23.

11. Da Silva Moreira J, Peixoto Camargo JD, et al. Lung abscess: analysis of 252 consecutive cases diagnosed between 1968 and 2004. J Brasil Pneumol 2008;32: 136-43.

12. Magalhaes L, Valadares D, Oliveira JR, Reis E. Lung abscesses. Review of 60 cases. Rev Port Pneumol 2009; 17:165-78

13. Sosenko A, Glassroth J. Fiberoptic bronchoscopy in the evaluation of lung abscesses. Chest 1985;87:489-94.

14. Mansharanani N, Balachandran D, Delaney D. Lung abscess in adults: clinical comparison of immunocompromised to non-immunocompromised patients. Resp Med 2002;96:178-85.

15. Fernandez-Sabe N, Carratala' J, Dorca J. Efficacy and safety of sequential amoxycillin-clavulanate in the treatment of anaerobic lung infections. Eur J Microbiol Infect Dis 2003;22:185-7. 